\title{
Himen Complaciente y Peritaje Médico-Legal
}

\author{
Dilatable or Complacent Hymen Legal Medical Expert Work
}

*,** Oscar Tapia E. \& *** Néstor San Martín U.

TAPIA, E. O. \& SAN MARTíN, U. N. Himen complaciente y peritaje médico-legal. Int. J. Morphol., 29(4):1435-1437, 2011.

RESUMEN: Se define como himen complaciente (HC) aquel himen que se distiende sin dañarse durante la penetración al momento de la relación sexual, por lo que no quedan lesiones atribuibles al acto sexual. Su hallazgo al momento de realizar el peritaje médico-legal impide afirmar la existencia de penetración total o parcial en el acto denunciado. El objetivo de este trabajo es determinar la prevalencia de HC junto con analizar variables socio-bio-demográficas en mujeres que denuncian ser víctima de una agresión sexual. Se realizó un estudio retrospectivo-descriptivo, seleccionándose de la Unidad de Sexología Forense del Servicio Médico Legal de Temuco las denuncias por violación realizadas entre Enero/2000-Diciembre/2003 ( $\mathrm{n}=509)$. Los datos se registraron en planilla Excel y analizados con el software Winstat para Excel. Se verificó 46 mujeres con HC (9\%). El 74\% de las víctimas con HC pertenecía al área urbana, siendo el promedio de edad 16,7 años. El 51,2\% denunció múltiples violaciones y el 89,2\% de los imputados resultaban ser conocidos de la víctima. Al examen físico se constató lesiones extragenitales y coloproctológicas en el 29,9\% y 4,4\% respectivamente. Dentro de las pruebas biológicas realizadas, en el 8,7\% se evidenció la presencia de espermios. En el 9\% de las mujeres que denuncian ser víctima de violación se constata HC. Al examen físico se encuentra algún tipo de lesión en sólo el 28,3\%, mientras que en el 8,7\% se demuestra la presencia de espermios. Con estos resultados son escasas las evidencias que permiten al médico sugerir la existencia de un atentado sexual con o sin penetración total o parcial.

PALABRAS CLAVE: Himen Complaciente; Delito sexual; Peritaje Médico-Legal.

\section{INTRODUCCIÓN}

El himen es una membrana de tipo mucoso que cierra parcialmente el introito vaginal, separando la vagina de la vulva (Testut \& Latarjet, 1954). Esta estructura presenta un doble origen embriológico, endodermo y seno urogenital, perforándose su orificio en la mayoría de los casos en el período fetal tardío (Hib, 1981). Es importante mencionar que la integridad del himen es considerado desde la antigüedad como sinónimo de virginidad (Testut \& Latarjet).

En la literatura se describen múltiples clasificaciones de tipos de himen, sin embargo, la mayoría de los autores coincide en que se identifican himen desflorados y no desflorados, pudiendo estos variar en su forma, tamaño orificial y elasticidad (Testut \& Latarjet; Jones et al., 2003; Berenson, 1995; Wang et al., 1995; Estel et al., 1995; Latoche \& Latoche, 1999). Según su elasticidad estos pueden ser dilatables o complacientes y dilatados, definiéndose como himen complaciente (HC) o dilatable a aquel himen que al examen ginecológico permite el paso de dos dedos enguantados sin romperse y al retirarlos vuelve a sus dimensiones normales o aquel que al momento de la relación sexual permite la penetración sin dañarse; conservando por tanto la virginidad anatómica (Latoche \& Latoche; Montoya \& Díaz, 2004). Por lo expuesto anteriormente, el hallazgo de un HC en una mujer que denuncia ser víctima de una violación, no le permite al médico afirmar la existencia de este delito, sino solo indicar su apreciación al respecto de acuerdo con las lesiones asociadas (Latoche \& Latoche; Montoya \& Díaz).

Con la finalidad de identificar la prevalencia de $\mathrm{HC}$ en las mujeres que denuncian en el Servicio Médico Legal de Temuco ser víctima de violación llevamos a cabo este trabajo, y así mismo caracterizar a este grupo según variables socio-biodemográficas.

\section{MATERIAL Y MÉTODO}

Se realizó un estudio retrospectivo-descriptivo, seleccionándose de los archivos de la Unidad de Sexología Forense del Servicio Médico Legal (SML) de Temuco las

* Departamento de Anatomía Patológica. Facultad de Medicina. Universidad de La Frontera. Temuco, Chile.

${ }^{* *}$ Scientific and Technological Bioresource Nucleus (BIOREN).

*** Médico Gineco-Obstetra, Perito Forense. Servicio Médico Legal, Temuco. 
denuncias por violación realizadas por mujeres entre Enero/2000 y Diciembre/2003.

Se definió como HC aquel himen que al momento de realizar el peritaje médico-legal permitió la introducción de dos dedos enguantados y lubricados sin dañarse o bien como aquel que se distendió hasta tres centímetros sin dañarse.

La recolección de datos se realizó a partir de un cuestionario protocolizado existente en la Unidad de Sexología Forense del SML de Temuco, en el cual se registran los procedimientos e información obtenida a partir de la aplicación de la normativa técnica pericial sobre agresiones sexuales del SML, la que incluye características socio-biodemográficas de las víctimas tales como: género, edad, área de residencia, estado civil y actividad de la víctima; del delito denunciado, del examen físico y de los resultados de pruebas de laboratorio.

Los datos se registraron en planilla Excel y analizados con el software Winstat para Excel.

\section{RESULTADOS}

De un total de 509 mujeres que denunciaron ser víctima de una violación en el período e institución antes señalado se constató al examen físico la presencia de $\mathrm{HC}$ en el $9 \%$ (46 casos).

La edad promedio del grupo estudiado fue 16,7 años (11-43 años), perteneciendo al área urbana el 74\% de la muestra analizada. Al momento de la agresión un 95,6\% (44) de las mujeres con HC era soltera y un 9\% (4) pertenecía a la etnia mapuche.

La actividad más frecuente de las víctimas al momento del atentado sexual era estudiante $(80,4 \%)$, concentrándose el 84,7\% de la muestra entre los 10 y 20 años. En cuanto a la periodicidad del atentado este fue único en $43 \%$ (20) y múltiples en un 56\% (26), siendo el agresor conocido por la víctima en el $89 \%$ de los casos denunciados; estando un familiar involucrado en el 69\% de estas agresiones. La distribución se detalla en la Tabla I.

Al momento de realizar el peritaje médico-legal se constataron lesiones extragenitales y coloproctológicas en el $28 \%$ y $4 \%$ respectivamente, comprobándose en el examen microscópico de la muestra de fluídos la presencia de espermios en el $9 \%$ de los casos.
Tabla I. Relación Víctima-Imputado.

\begin{tabular}{lclc}
\hline Relación Víctima-Imputado & $\%$ & & $\%$ \\
\hline \multirow{2}{*}{ Conocido } & & Familiar Padre & 24 \\
& 89 & $\begin{array}{l}\text { Familiar No Padre } \\
\text { No Familiar }\end{array}$ & 45 \\
Desconocido & & & 20 \\
No Aportado & 9 & & \\
\hline
\end{tabular}

\section{DISCUSIÓN}

Se constató la presencia de $\mathrm{HC}$ en un $9 \%$ de las mujeres que denunciaron ser víctimas de una violación entre Enero/2000-Diciembre/2003 en el SML de Temuco, cifra bastante inferior a lo publicado en otras series internacionales, no existiendo, sin embargo; literatura nacional al respecto (Latoche \& Latoche).

$\mathrm{Al}$ igual que lo reportado por Kar et al. (2010) en nuestro trabajo la mayoría de los imputados $(89 \%)$ correspondía a sujetos conocidos de la víctima (familiares o amigos).

El hallazgo de un HC acompañado de la ausencia de lesiones extragenitales, coloproctológicas y de espermios en un alto porcentaje de los casos (68\%); determinan que un grupo de mujeres que denuncian ser víctima de una agresión sexual no presenten lesiones atribuibles a este delito, dificultando por tanto el peritaje que permitan al médico legista sugerir la existencia de un atentado sexual con o sin penetración vaginal ya sea parcial o total, siendo de vital importancia para mejorar el rendimiento de este procedimiento el tiempo transcurrido entre el atentado sexual y la realización del examen físico con toma de muestras (Jones et al.; Kar et al.; Christian et al., 2000; Lauritsen et al., 2000).

Por las razones expuestas anteriormente, la investigación del delito denunciado no se debe basar únicamente en torno a la presencia o no de lesiones himeneales, sino que deben conjuntamente considerarse los peritajes de otros profesionales tales como peritos psiquiatras, psicólogos, de Carabineros y de la Policia de Investigaciones (Latoche \& Latoche; Montoya et al.; Bond et al., 1995; Berenson, 1994).

Cabe mencionar que los casos estudiados no corresponden técnicamente a una muestra real de la población víctima de atentados sexuales, puesto que la totalidad de dicha población es desconocida y con seguridad mucho más numerosa, ya que toda la información existente para la realización de estudios se basa en la denuncia voluntaria por parte de las víctimas o sus familiares, y lo que según diversos 
autores no va más allá de un $20 \%$ de todas las agresiones sexuales, quedando aproximadamente un $80 \%$ de estos delitos sin denuncia y por lo tanto no sujeta a análisis (Woodling et al., 1977; González et al., 1997; San Martín et al., 2003).

TAPIA, E. O. \& SAN MARTÍN, U. N. Dilatable or complacent hymen, legal medical expert work. Int. J. Morphol., 29(4):14351437, 2011.

SUMMARY: Dilatable or complacent hymen is defined as that which can be stretched without being damaged during sexual intercourse, and is the reason as to lack of injuries present that are attributable to the sexual act. The findings at the time the legal medical expert work is carried out, cannot confirm the existence of total or partial penetration in the denounced cases. The objective of this study was to identify the prevalence of dilatable or complacent hymens and analyze the social-bio-demographic factors of the victims of sexual assault. A descriptive, retrospective study was carried out selecting the reports of rapes from the Sexology Unit of the Legal Medical Service in the city of Temuco, between January 2000 and December $2003(n=509)$. The information was registered with Excel program and analyzed by Winstat Software for Excel program. Dilatable or complacent hymen in 46 cases (9\%), $74 \%$ of the victims were from urban areas, with an average of 16.7 years of age. $51.2 \%$ reported several episodes and $89.2 \%$ of the aggressors were individuals familiar to the victim. During physical examination extra genital and colorectal injuries were noted in $29.9 \%$ and $4.4 \%$ respectively. During biological exams presence of spermatocytes was confirmed in $8.7 \%$. Dilatable or complacent hymen was reported in $9 \%$ of the rapes. During physical examination evidence of injury is reported only in $28.3 \%$, while there is evidence of spermatocytes in $8.7 \%$ of the victims. As result there is little evidence that allows the physician to determine the existence of a sexual assault with or without penetration.

KEY WORDS: Dilatable or complacent hymen; Sexual aggression; Legal medical expert work.

\section{REFERENCIAS BIBLIOGRÁFICAS}

Berenson, A. B. The prepubertal genital exam: what is normal and abnormal. Curr. Opin. Obstet. Gynecol., 6(6):526-30, 1994.

Berenson, A. B. A longitudinal study of hymenal morphology in the first 3 years of life. Pediatrics, 95(4):490-6, 1995.

Bond, G. R.; Dowd, M. D.; Landsman, I. \& Rimsza, M. Unintentional perineal injury in prepubescent girls: a multicenter, prospective report of 56 girls. Pediatrics, 95(5):628-31, 1995.

Christian, C. W.; Lavelle, J. M.; De Jong, A. R.; Loiselle, J.; Brenner, L. \& Joffe, M. Forensic evidence findings in prepubertal victims of sexual assault. Pediatrics, 106(1 Pt 1):100-4, 2000.
Estel, C.; Baum, M. \& Tausch, S. Diagnosis and therapy of imperforate hymen. Zentralbl. Gynakol., 117(2):108-10, 1995.

González, E.; Molina, T.; Toledo, V.; Luengo, X.; Molina, C. \& Molina, R. Violación intra y extrafamiliar en adolescentes: variables personales y familiares seleccionadas. Rev. Chil. Obstet. Ginecol. Infant. Adolesc., 4(1):13-21, 1997.

Hib, J. Embriología médica. $2^{\text {a }}$ ed. Buenos Aires. El Ateneo, 1981. p. 241

Jones, J. S.; Rossman, L.; Wynn, B. N.; Dunnuck, C. \& Schwartz, N. Comparative analysis of adult versus adolescent sexual assault: epidemiology and patterns of anogenital injury. Acad. Emerg. Med., 10(8):872-7, 2003.

Kar, H.; Arslan, M.; Çekin, N.; Akçan, R. \& Hilal, A. Sexual Assault in Childhood and Adolescence; a Survey Study. Eur. J. Soc. Sci., 13(4):549-55, 2010.

Latoche, E. \& Latoche, C. Estudio anatómico del himen y su repercusión en medicina legal. Med. Leg. Costa Rica, 16(1-2):27, 1999.

Lauritsen, A. K.; Meldgaard, K. \& Charles, A. V. Medical examination of sexually abused children: medico-legal value. J. Forensic Sci., 45(1):115-7, 2000.

Montoya, S. D.; Díaz, S. R.; Reyes, O. F.; Abusleme, A. C. \& Garrido, C. J. Peritaje médico legal en delitos sexuales: una pauta practica para su correcta realización. Rev. Chil. Obstet. Ginecol., 69(1):55-9, 2004.

San Martín, N.; González, L.; Barrientos, V. \& Gutiérrez, R. Perfil epidemiológico de las víctimas y características generales de los atentados sexuales en la IX Región. Rev. Fronteras en Obstetricia y Ginecología, 3(1):11-7, 2003.

Testut, L. \& Latarjet, A. Tratado de anatomía humana. $9^{\mathrm{a}}$ ed. Madrid, Salvat, 1954. pp.1331-5. Tomo IV.

Wang, J.; Ezzat, W. \& Davidson, M. Transverse vaginal septum. A case report. J. Reprod. Med., 40(2):163-6, 1995.

Woodling, B. A.; Evans, J. R. \& Bradbury, M. D. Sexual assault: rape and molestation. Clin. Obstet. Gynecol., 20(3):509-30, 1977.

Dirección para correspondencia:

Dr. Oscar Tapia E.

Departamento de Anatomía Patológica

Facultad de Medicina, Universidad de La Frontera

Manuel Montt 112

Código Postal 478-1176, Temuco

CHILE

Email: otescalona@gmail.com

Recibido: 18-08-2011

Acepado: 22-09-2011 